\title{
Motor Recovery in the Absence of Segmental Afferents: A Case Study of Incomplete Spinal Cord Injury
}

\author{
J. W. Little, MD, PhD, ${ }^{1,2}$ R. M. Harris, PhD, ${ }^{3}$ D. Smithson, MD $^{2}$ \\ ${ }^{1}$ Spinal Cord Injury Service, Veterans Administration Medical Center, ${ }^{2}$ Depart- \\ ment of Rehabilitation Medicine, ${ }^{3}$ Department of Biological Structure, University \\ of Washington, Seattle, Washington, USA.
}

\begin{abstract}
Summary
A 41-year-old male with a prior left $L-5 / S-1$ radiculopathy developed complete quadriplegia following a gunshot wound to the left anterior neck. He subsequently recovered pinprick sensation over the left side of the trunk and lower extremity; and a right peroneal SEP, suggested sparing of long tracts on the right side. Voluntary motor strength gradually recovered more on the left than right for the $L-5 / S-1$ segments, where tendon reflexes were absent. This unique case is discussed with respect to the effect of absent segmental afferents on suprasegmental recovery.

Key words: Spinal cord injury; Motor recovery; Dorsal roots; Brown-Sequard syndrome; Recovery mechanisms.

Recovery of voluntary movement is common following incomplete spinal cord injury. When spinal long tracts are spared more on one side than the other, as in the Brown-Sequard syndrome, more voluntary strength returns on the side with the most sparing (Taylor and Gleave, 1957; Nathan and Smith, 1973; Little and Halar, 1985). This asymmetrical recovery is attributed to a greater number of spared descending motor axons on one side of the spinal cord (Nathan and Smith, 1973; Little and Halar, 1985). This paper describes the reverse pattern, greater motor recovery on the side with least sparing of spinal cord long tracts, in a patient who had prior lumbosacral radiculopathy and absent tendon reflexes on that side. This unique case provides a natural experiment in which segmental afferents have been removed, and their role in spinal cord motor recovery can be examined.
\end{abstract}

Correspondence to: J. W. Little SCI (128), Veterans Administration Medical Center, 1660 S. Columbian Way, Seattle, WA 98108, USA. 
Table I Clinical exam at 10 months post-injury

\begin{tabular}{lccc}
\hline \multicolumn{1}{c}{\begin{tabular}{c} 
Muscle strength \\
\multicolumn{1}{c}{$(0-5$ scale $)$}
\end{tabular}} & $\begin{array}{c}\text { Segmental } \\
\text { innervation }\end{array}$ & Right & Left \\
\hline Hip flexion & L-1/L-3 & $2+$ & 1 \\
Knee extension & L-2/L-4 & $3-$ & $3-$ \\
Hip extension, gluteus maximus & L-5/S-2 & 0 & $2-$ \\
Knee flexion & L-5/S-2 & $2-$ & 2 \\
Ankle dorsiflexion & L-4/L-5 & $2-$ & $2+$ \\
Ankle plantarflexion & L-5/S-2 & $2+$ & $4-$ \\
Extensor hallucis longus & L-5/S-1 & 0 & $3+$ \\
Toe (2-5) plantarflexion & L-5/S-1 & 1 & $4-$ \\
Tendon reflexes $(0-4$ scale $)$ & & & 3 \\
Patellar reflex & L-2/L-4 & 3 & 0 \\
Medial Hamstring reflex & L-5 & 2 & 0 \\
Achilles reflex & S-1 & 4 & \\
Sensation in lower extremities & & & Present but impaired \\
Pinprick & & Absent & Absent \\
Temperature & & Absent & Absent \\
Position-sense, toe & & Absent & Absent \\
Position-sense, ankle & & Present & Absent \\
Position-sense, knee & & Absent & \\
\hline
\end{tabular}

Table II Electrodiagnostic studies

\begin{tabular}{lcc}
\hline & Right & Left \\
\hline Tibial H-reflex, amplitude/latency & $7.5 \mathrm{mV} / 37 \cdot 0 \mathrm{msec}$ & Absent \\
Tibial M-response, amplitude/latency & $8.8 \mathrm{mV} / 6.2 \mathrm{msec}$ & $6.2 \mathrm{mV} / 5.4 \mathrm{msec}$ \\
Somatosensory evoked potential & & \\
Peroneal nerve & Present $(\mathrm{N} 1=47.9 \mathrm{~ms})$ & Absent \\
Femoral & Absent & Absent \\
Saphenous & Absent & Absent \\
\hline
\end{tabular}

\section{Case report}

A 41-year-old male with a prior history of left lumbosacral root damage was shot in the left anterior neck during an altercation, and has been followed for 10 months post-injury. The bullet passed through the left C-7 pedicle and lamina, sparing the spinal canal. $\mathrm{He}$ was initially paralysed in all extremities and anesthetic below C-8. Sensation returned in the trunk and lower extremities, with some sharp-dull discrimination present on the left but not on the right, and some joint position sense on the right but not on the left (Table I). Temperature and vibratory sensation have not returned. An SEP was recorded to right but not left peroneal nerve stimulation (DeLisa et al., 1987). SEPs were absent to femoral and saphenous nerve stimulation bilaterally (Table II).

Initially, both upper extremities were profoundly weak in C-8/T-1 innervated muscles. Right/Left grip strength gradually improved from 10/1 pounds at 3 weeks to $102 / 35$ pounds at 6 months post-injury, the greater strength on the right being consistent with the asymmetric cord injury.

Voluntary movements were first noted on day 9 in the left foot as trace toe extension and ankle plantarflexion. Shortly thereafter, trace knee extension and toe/foot plantarflexion were noted on the right. Recovery of these voluntary movements was accompanied by the appearance of hypertonus, particularly in hip adductors and knee extensors bilaterally. By 10 months post-injury, voluntary strength had gradually 
A

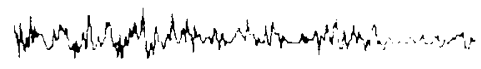

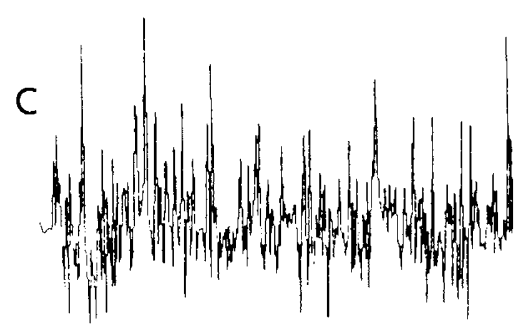

B

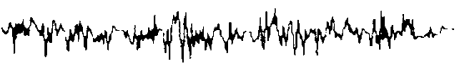

D

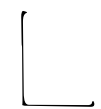

Figure Voluntary recruitment of leg muscles: Rectus femoris, left (A) and right (B) during maximal resisted straight-leg raising; Gastrocnemius, left $(C)$ and right (D) during maximal resisted plantarflexion of the ankle. Note the greater activity in left than right gastrocnemius. Horizontal scale $=1 \mathrm{sec} /$ division; vertical scale $=100 \mathrm{uV} /$ division for $(A)$ and $(B)$, $200 \mathrm{uV} /$ division for (C) and (D).

improved. In muscles innervated by lumbar segments L-2 to L-4, right lower extremity strength recovered as much or more than did the left. However, in muscles innervated by L-5 and S-1 segments, left sided strength recovered more than right (Table I).

The motor recovery at different cord levels was confirmed with electromyographic recordings (Figure). Surface recordings revealed greater voluntary recruitment in the right rectus femoris (L-2/L-4) as compared to the left, but greater left gastrocnemiussoleus recruitment (L-5/S-1) than right. No antagonist electromyographic activity was recorded in the right tibialis anterior nor in the right gastrocnemius-soleus muscles with voluntary ankle plantarflexion or dorsiflexion respectively. Needle EMG recordings demonstrated slow maximal firing rates of 5 to $10 \mathrm{~Hz}$ in all right and left lower extremity muscles. Motor-unit action potentials were of normal amplitude and shape with few or no positive sharp waves or fibrillations. This is consistent with weakness due to an upper motorneuron lesion.

Patellar, Achilles and medial hamstring tendon reflexes were low-threshold and large amplitude except that the left Achilles (S-1) and left medial hamstring (L-5) tendon reflexes were absent. The patient stated that his left Achilles reflex had been absent since the onset of left back and leg pain 7 years earlier, for which he had undergone L-5 laminectomy and discectomy. Electrodiagnostic studies, done after the cervical gunshot wound (Table II), confirmed the absence of the left tibial H-reflex, which is mediated by the $S-1$ segmental reflex arc. The left gastrocnemius-soleus compound muscle action potential (M-response) to tibial nerve stimulation was, however, of large amplitude and normal latency. The absence of the left Achilles tendon reflex is thus due to disruption of the afferent but not the efferent root. The right tibial $\mathrm{H}$-reflex and $\mathrm{M}$-response were of normal latency and amplitude. 


\section{Discussion}

In this patient, the bullet passed adjacent to the left side of the cervical spinal cord, indirectly damaging spinal long tracts (similar to Klemperer et al., 1954) and causing an initial complete paralysis of the lower extremities. The partial motor recovery which followed was likely mediated by spared motor pathways descending on the right side of the spinal cord, as described for other cases of Brown-Sequard syndrome (Nathan and Smith, 1973; Little and Halar, 1985). The pattern of sensory and motor return is consistent with this explanation, except at the L-5/S-1 levels where return of voluntary motor strength was unexpectedly greater on the left. The patient's absent left L-5/S-1 reflexes, due to prior dorsal root damage from a disc herniation, suggest an explanation for this unusual recovery pattern.

We believe that compromised left L-5 and S-1 afferents have allowed enhanced recovery of voluntary strength. Segmental inhibitory influences exist, both as pre-synaptic and post-synaptic inhibition; perhaps such inhibition limits recovery of voluntary movement (Robinson et al., 1986). In our patient, perhaps the loss of L-5 and S-1 dorsal root afferents has reduced segmental inhibition and thus allowed greater recovery of voluntary strength.

A second possible mechanism is that hyperactive segmental stretch reflexes limit voluntary movement because of antagonist muscle co-contractions (Yanagisawa et al., 1976; Knuttson, 1983). In this patient, however, surface EMG recordings showed that antagonist co-contraction was not present in the right gastrocnemius-soleus muscle during active ankle dorsiflexion, nor in the right tibialis anterior muscle during active ankle plantarflexion.

A third mechanism that would explain a limiting effect by segmental afferents on suprasegmental voluntary recovery presupposes collateral sprouting and competitive synaptogenesis by the segmental reflex afferents and the spared descending axons. These segmental and suprasegmental inputs are postulated to mediate, respectively, the appearance of hyperreflexia and the recovery of voluntary movements following incomplete spinal cord injury (Liu and Chambers, 1958; Murray and Goldberger, 1974; Little, 1976). Synaptogenesis in response to partial denervation is reportedly competitive in the peripheral (Bixby and VanEssen, 1979; Ostberg and Vrbova, 1982; Liestol et al., 1985; Ribchester and Taxt, 1983) and in the central nervous system (Field et al., 1980). Following incomplete spinal cord injury, there may be competitive synaptogenesis between the segmental reflex afferents and the descending axons for denervated postsynaptic sites in the spinal cord. In our patient, reduced numbers of reflex afferents due to the prior radiculopathy may have allowed greater synaptogenesis by spared descending axons at the L-5/S-1 level, and thus greater recovery of voluntary strength.

Since such a competitive interaction between reflex afferents and descending input may explain the reciprocal relationship between motor recovery and hyperreflexia in this patient, animal studies would seem warranted to further explore the possibility of enhancing recovery of voluntary movement by limiting segmental afferent influences. 


\section{Acknowledgements}

Supported by the Rehabilitation Research and Development Service, Veterans Administration. We thank Paula Micklesen for assistance with manuscript preparation.

\section{References}

BIXBY JL, VANESSEN DC 1979 Competition between foreign and original nerves in adult mammalian skeletal muscle. Nature 282:726-728.

DeLisa JA, MACKenzie K, Baren EM 1987 Manual of Nerve Conduction Velocity and Somatosensory Evoked Potentials, 2nd Edn. Raven Press, New York.

Field PM, Coldham DE, RaIsman G 1980 Synapse formation after injury in the adult rat brain: preferential reinnervation of denervated fimbrial sites by axons of the contralateral fimbria. Brain Research 189:103-113.

Kendall FP, McCreary EK 1983 Muscle Testing and Function, 3rd Edn. Williams \& Wilkins, Baltimore.

KLEMPERER WW, Fulton JF, LAMPORT H, et al. 1954 Indirect spinal cord injuries due to gunshot wounds of the spinal column in animal and man. The Military Surgeon 114:253-265.

KNUTTSON E 1983 Analysis of gait and isokinetic movements for evaluation of antispastic drugs or physical therapies. In: Desmedt JE (ed) Motor Control Mechanisms in Health and Disease, Raven Press, New York, pp 1013-1034.

Liestol K, MACHLER J, NJA A 1986 Selective synaptic connection: significance of recognition and competition in mature sympathetic ganglia. Trends in Neuroscience 9(1):21:24.

LitTle JW 1976 Recovery of Function Following Spinal Cord Lesions in Cats. PhD Dissertation, University of Chicago, Chicago.

LITTLE JW, HALAR E 1985 Temporal course of motor recovery after Brown-Sequard spinal cord injuries. Paraplegia 23:39-46.

LiU C-N, Chambers WW 1958 Intraspinal sprouting of dorsal root axons. Archives of Neurology and Psychiatry 79:46-61.

MURRAY M, GoldBERGER ME 1975 Restitution of function and collateral sprouting in the cat spinal cord: the partially hemisected animal. Fournal of Comparative Neurology 158:19-36.

NATHAN PW, SMITH MC 1973 Effects of two unilateral cordotomies on the motility of the lower limbs. Brain 96:471-494.

OStberg A, VRobova G 1982 Competitive reinnervation of the rat superior cervical ganglion by foreign nerves. Neuroscience 7:3177-3189.

RIBCHESTER RR, TAXT T 1983 Motor unit size and synaptic competition in rat lumbrical muscle reinnervated by active and inactive motor axons. Fournal of Physiology 344:89-111.

ROBINSON GA, GOLDBERGER ME 1985 Interfering with inhibition may improve motor function. Brain Research 346:400-403.

TAYLOR RG, GLEAVE JRW 1957 Incomplete spinal cord injuries with Brown-Sequard phenomena. Fournal of Bone and foint Surgery 39B:438-450.

Tanagisawa N, TANAKa R, Ito Z 1976 Reciprocal Ia inhibition in spastic hemiplegia of man. Brain 99:555-574. 